\title{
Intraepithelial Neoplasia
}

National Cancer Institute

\section{Source}

National Cancer Institute. Intraepithelial Neoplasia. NCI Thesaurus. Code C8366.

A precancerous neoplastic process that affects the squamous, glandular, or transitional cell epithelium without evidence of invasion. According to the degree of nuclear atypia, number of mitotic figures, and presence of architectural distortion, it is classified as low grade (mild dysplasia) or high grade (moderate or severe dysplasia). 